\title{
Caractéristiques de la filtration capillaire pulmonaire pendant une augmentation du débit cardiaque associée à une vasodilatation
}

\author{
Sagazaga Drissa SANOU ${ }^{1 *}$, Michel CORBOZ ${ }^{2}$, Gila BENCHETRIT ${ }^{2}$, Daniel \\ ANGLADE $^{2}$ et Francis GRIMBERT ${ }^{2}$ \\ ${ }^{1}$ Département de Physiologie Animale et de Biologie Animale, UFR/SVT de l'Université de Ouagadougou, 10 \\ BP 13894 Ouagadougou 10 Burkina Faso. \\ ${ }^{2}$ Laboratoire TIMC-IMAG, UMR Université Joseph Fourier-CNRS 5525, 38700 la Tronche, France. \\ *Auteur correspondant,E-mail: dsanou@univ-ouaga.bf, sanoudrissa82@yahoo.fr
}

\section{RESUME}

Les effets du débit cardiaque sur la filtration capillaire pulmonaire (FCP) diffèrent selon que le débit cardiaque est augmenté mécaniquement, entraînant une augmentation du débit lymphatique pulmonaire (Q1) et une baisse du rapport de la concentration protéique de la lymphe sur celle du plasma $(\mathrm{Cl} / \mathrm{Cp})$, ou par l'exercice où $\mathrm{Ql}$ augmente sans diminution significative de $\mathrm{Cl} / \mathrm{Cp}$. Afin d'expliquer ces différences, nous avons émis l'hypothèse qu'une vasodilatation était associée à l'exercice. Afin de vérifier cette hypothèse chez le chien anesthésié, nous avons perfusé un vasodilatateur le nitroprussiate de sodium, en association à une augmentation mécanique de débit cardiaque par l'ouverture de shunts artério-veineux. Nous avons comparé les résultats hémodynamiques et les variables lymphatiques à ceux d'une étude précédente où seule une augmentation mécanique de débit cardiaque avait été réalisée. Ql a été multiplié par deux, mais $\mathrm{Cl} / \mathrm{Cp}$ n'a pas varié significativement. En conclusion, l'association d'un vasodilatateur à l'augmentation mécanique de Qc rapproche les résultats de notre étude de ceux observés dans d'autres études à l'exercice. Ces résultats sont en faveur d'un recrutement et d'une augmentation de la surface d'échange capillaire pulmonaire liée à la libération d'oxyde nitrique par l'endothelium capillaire pulmonaire stimulé par l'augmentation du débit sanguin pulmonaire pendant l'exercice.

(C) 2010 International Formulae Group. All rights reserved.

Mots clés: Circulation pulmonaire, Débit sanguin pulmonaire chez le chien, Perméabilité capillaire, Nitroprussiate de sodium, Système lymphatique.

\section{INTRODUCTION}

Le liquide et les protéines filtrés au travers des parois capillaires pulmonaires sont drainés par la circulation lymphatique pulmonaire vers les veines de la circulation systémique. Ce drainage permet d'éviter l'accumulation du filtrat et un odème dans les espaces extravasculaires pulmonaires. Le débit $(\mathrm{Ql})$ et la richesse en protéines de la lymphe pulmonaire mesurée par la concentration en protéines de la lymphe rapportée à celle du plasma $(\mathrm{Cl} / \mathrm{Cp})$ peuvent être utilisés pour estimer la filtration capillaire pulmonaire (FCP) dans les préparations expérimentales in vivo, notamment chez le mouton et le chien. L'augmentation de Q1 est une mesure très sensible d'une élévation de la FCP avant que le drainage lymphatique ne soit 
débordé et que l'eau extravasculaire n'augmente, signant l'apparition de l'oedème pulmonaire. Afin de mesurer la perméabilité transcapillaire, il est habituel de tester les parois capillaires à l'aide d'une augmentation de la pression capillaire pulmonaire $(\mathrm{Pc})$. L'augmentation de Pc est habituellement obtenue dans ce type de préparation par une augmentation de la pression à la sortie de la circulation pulmonaire, en pratique par une augmentation de la pression dans l'oreillette gauche $(\mathrm{Pog})$. Si la perméabilité des parois capillaires est intacte, la filtration augmentera ainsi que le tamisage des protéines au travers des parois capillaires. Il en résultera une augmentation de Q1 et une baisse de $\mathrm{Cl} / \mathrm{Cp}$ aussi bien chez le mouton (Erdmann et al., 1975), que chez le chien (Parker et al., 1981; Stewart et al., 2004). Si la perméabilité des parois capillaires pulmonaires est augmentée, il en résultera une augmentation de Q1 avec un rapport $\mathrm{Cl} / \mathrm{Cp}$ qui restera stable voire augmentera (Grimbert et al., 1981; Jonkam et al., 2009). Il est à noter qu'un recrutement de capillaires dont les caractéristiques de filtration seront similaires, avec l'augmentation de la surface d'échange capillaire qui lui est liée, entraînera également une augmentation de $\mathrm{Ql}$ avec un rapport $\mathrm{Cl} / \mathrm{Cp}$ qui demeurera peu abaissé voire stable. Un tel recrutement peut survenir lorsque le débit cardiaque (Qc) est augmenté, lorsque Pc est augmentée, ou lors d'une vasodilatation artérielle de la circulation pulmonaire.

Les études rapportant les effets d'une augmentation mécanique de Qc sur les caractéristiques de la FCP d'un poumon normal perfusé en zone 3 (Pap > Pog > P aérienne alvéolaire) (West et al., 1964) donnent des résultats assez homogènes quelles que soient les techniques utilisées pour augmenter mécaniquement Qc. Il est observé une augmentation de Q1 avec abaissement du rapport $\mathrm{Cl} / \mathrm{Cp}$, avec un tamisage des protéines similaire à celui observé lorsque l'on élève Pc par élévation de la pression de l'oreillette gauche (Feltes et al., 1989; Grimbert et al., 1998; Landolt et al., 1984). Les études rapportant les effets d'une augmentation de Qc à l'exercice sur les caractéristiques de la FCP d'un poumon normal perfusé en zone 3 font état de résultats plus variables, soit une augmentation de Q1 associée à un abaissement de $\mathrm{Cl} / \mathrm{Cp}$, soit une augmentation de $\mathrm{Ql}$ avec un abaissement du rapport $\mathrm{Cl} / \mathrm{Cp}$ faible ou absent évoquant une augmentation de la surface d'échanges (Coates et al., 1984, Koizumi et al., 2001; Newmann et al., 1988; Newman et al., 1993; O'brodowith et al., 1986).

Nous avons fait l'hypothèse que ces différences de caractéristiques de FCP pouvaient être liées à l'existence d'une vasodilatation vasculaire pulmonaire d'origine neuro-humorale contemporaine de l'exercice, vasodilatation qui ne serait pas présente lorsque Qc est augmenté mécaniquement. Afin de vérifier cette hypothèse, nous avons augmenté Qc grâce à des shunts artérioveineux et nous $y$ avons associé une vasodilatation pharmacologique par perfusion du nitroprussiate de sodium (expériences augm Qc + NP). Nous avons ensuite comparé nos résultats à ceux obtenus dans une étude précédente de ce laboratoire où une augmentation mécanique de Qc (expériences augm Qc) avait été réalisée sur la même préparation expérimentale (Grimbert et al., 1988). Enfin, nous avons vérifié si les résultats des expériences (augm Qc + NP) se rapprochaient davantage que ceux des expériences augm Qc de ceux des études ayant mesuré la FCP à l'exercice.

\section{MATERIEL ET METHODES}

\section{Anesthésie, mesures hémodynamiques, shunts artério-veineux, canulation lymphatique pulmonaire}

Quatorze chiens pesant entre 11 et 28 $\mathrm{kg}$ ont été utilisés pour l'ensemble de ces expériences. Ils sont anesthésiés par injection 
intraveineuse de thiopental sodique à raison de $35 \mathrm{mg}$ par $\mathrm{kg}$ de poids. Ils sont ensuite équipés d'une sonde d'intubation endotrachéale qui permet de les ventiler avec une pompe (Palmer) à une fréquence de 24 cycles par minute. Le volume courant de 15 $\mathrm{ml}$ par $\mathrm{kg}$. Une pression expiratoire positive égale à $3 \mathrm{~cm}$ d'eau est appliquée afin d'éviter toute atélectasie pulmonaire. La température centrale est mesurée en continue par une thermistance introduite dans l'œsophage.

Sont ensuite mis en place :

- par dénudation de l'artère fémorale, un cathéter destiné à mesurer la pression artérielle systémique (Pas) et à prélever les échantillons de sang pour mesurer les gaz du sang $(\mathrm{PaO} 2, \mathrm{PaCO} 2, \mathrm{pH})$;

- par voie percutanée, un cathéter veineux périphérique qui sert à l'injection à la demande de la substance anesthésique et la perfusion de sérum glucosé isotonique. Un cathéter de Swan-Ganz est introduit dans une veine fémorale par dénudation pour la mesure de la pression artérielle pulmonaire (Pap), de la pression artérielle pulmonaire d'occlusion, et du débit cardiaque (Qc) par thermodilution. Ce cathéter permet également d'estimer la pression capillaire pulmonaire $(\mathrm{Pc})$ par une méthode dérivée de celle d'Holloway (Holley et al., 1983).

En plus des cathéters précédents, un shunt de gros calibre est installé entre l'artère carotide et la veine jugulaire gauche. A ce shunt de gros calibre, un deuxième de plus petit calibre est installé entre l'artère et la veine fémorale.

Une fois équipé des différents cathéters, l'animal est placé en décubitus latéral droit et une thoracotomie est pratiquée dans le sixième ou septième espace intercostal gauche. Cette approche permet :

- d'introduire une sonde à ballonnet dans l'oreillette gauche afin de mesurer la pression de l'oreillette gauche (Pog) et éventuellement de gonfler le ballonnet pour augmenter la Pog dans le cas où l'on veut tester les capacités de tamisage aux protéines de la paroi capillaire pulmonaire (Parker et al., 1981)

- d'accéder au site de canulation lymphatique. Les poumons sont réclinés afin de dégager la région circonscrite par la crosse de l'aorte et l'artère pulmonaire. Les vaisseaux lymphatiques sont colorés par l'instillation, juste avant la thoracotomie, au niveau de la bifurcation trachéale, d'environ $2 \mathrm{ml}$ d'une solution à $2 \%$ de bleu Evans. Un vaisseau lymphatique afférent au ganglion trachéobronchique gauche est dégagé et ligaturé du côté céphalique. Une aiguille prolongée par un fin tuyau est introduite dans le vaisseau lymphatique et ligaturée sur celuici.

La concentration des protéines de la lymphe et du plasma est mesurée par réfractométrie.

\section{Protocole expérimental}

Le protocole expérimental, illustré dans la Figure 1, commence par un état stable de contrôle de 2 heures en moyenne pendant laquelle sont mesurés toutes les 15 minutes $\mathrm{Q} 1,(\mathrm{Cl})$, les variables hémodynamiques (Pas, Pap, Pc, Pog, Qc, fréquence cardiaque) et la température ; toutes les 30 minutes, un échantillon de sang veineux est prélevé pour mesurer l'hématocrite et $\mathrm{Cp}$. Ensuite se fait l'augmentation de Qc réalisée par l'ouverture des shunts artério-veineux couplée à une vasodilatation par perfusion de nitroprussiate de sodium (1 $\mathrm{mg} / \mathrm{ml})$. Le débit de nitroprussiate de sodium (NP) est réglé de telle sorte que Pas diminue de $20 \%$ par rapport à sa valeur de contrôle. Quand cet équilibre est atteint, les différentes variables sont mesurées comme durant 2 à 3 heures jusqu'à obtenir un état stable. Ce dernier est obtenu lorsque les valeurs des mesures se stabilisent pendant $45 \mathrm{mn}$ à une heure.

Afin de vérifier l'absence d'anomalies 
de la perméabilité capillaire qui pourraient fausser les résultats des préparations expérimentales, nous avons réalisé des stimuli hydrostatiques en augmentant la Pog. Ces stimuli étaient obtenus par gonflement du ballonnet dans l'oreillette gauche pendant 2 à 3 heures. Le but de ces stimuli était de tester la qualité de la paroi capillaire, c'est-à-dire sa capacité de tamisage des protéines. Ces stimuli hydrostatiques étaient réalisés en deux occasions :

- 4 expériences témoins où les shunts artério-veineux n'ont pas été ouverts et où le NP n'a pas été perfusé,

- un état stable supplémentaire dans 3 expériences où nous avons élevé la Pog après avoir atteint un état stable d'élévation du Qc et de perfusion de NP. Les shunts artérioveineux étaient alors fermés et la perfusion de NP arrêtée avant d'élever la Pog.

\section{Analyses statistiques}

Les valeurs des variables hémodynamiques et lymphatiques ont été évaluées en calculant les moyennes $\pm \mathrm{SE}$. Ces valeurs ont été comparées par analyse de variance entre l'état de contrôle et l'état de stimulation. La significativité est déterminée par le test de Student avec $\mathrm{p}<0,05$.

\section{RESULTATS}

Modifications hémodynamiques et lymphatiques induites par l'augmentation du débit cardiaque associée à la vasodilatation par le nitroprussiate de sodium (augm Qc + NP)

Les valeurs hémodynamiques moyennes $(\mathrm{n}=10)$ du deuxième état stable (augm Qc+NP) sont exposées après l'état stable de contrôle dans le Tableau 1. La Pas est abaissée de $20 \%$ par la perfusion du NP conformément au protocole. L'ouverture des shunts artério-veineux associée à la perfusion de NP élève Qc de 1,97 $\pm 0,151 /$ min à 2,84 \pm $0,31 / \min$ ( $p<0,05$ ), soit $44 \%$ en moyenne. La
Pap $(20,6 \pm 1,4 \mathrm{~mm} \mathrm{Hg}$ vs. $18,9 \pm 1,2 \mathrm{~mm} \mathrm{Hg})$ est légèrement augmentée mais non significativement. La Pog reste stable à $6 \pm$ $0,8 \mathrm{~mm} \mathrm{Hg}$. La Pc augmente de 13,6 11 à $15,5 \pm 1 \mathrm{~mm} \mathrm{Hg}(\mathrm{P}<0,05)$. On note une diminution significative des résistances vasculaires pulmonaires (RVP) qui passent de $3,95 \pm 0,94$ à $3,10 \pm 0,73 \mathrm{~mm} \mathrm{Hg} 1^{-1} \min (\mathrm{p}<$ $0,05)$.

Les valeurs biologiques sont dans les limites de la normale et n'ont pas été significativement modifiées par rapport aux valeurs de l'état stable de contrôle. La $\mathrm{PaO} 2$ (pression partielle d'oxygène du sang artériel) est de 14,4 $\pm 1,6 \mathrm{kPa}$, la $\mathrm{PaCO} 2$ (pression partielle du gaz carbonique du sang artériel) est de 4,84 $\pm 0,27 \mathrm{kPa}$, le pHa du sang artériel est de 7,35 $\pm 0,02$. L'hématocrite est de 45,9 $\pm 0,01 \%$.

Les valeurs moyennes des variables lymphatiques du deuxième état stable (augm Qc+NP) sont exposées après l'état stable de contrôle dans le Tableau 1. Q1 atteint 30,1 \pm 7,5 vs. $16,0 \pm 4,9 \mu \mathrm{l} / \mathrm{min}$ soit à peu près le double de sa valeur de contrôle $(\mathrm{p}<0,01)$. $\mathrm{Cl}$ est peu modifiée mais $\mathrm{Cp}$ est abaissée de 62,0 $\pm 2,3$ à 54,5 $\pm 5,7 \mathrm{~g} / \mathrm{l},(\mathrm{p}<0,05)$. Nous avons attribué cette diminution de $\mathrm{Cp}$, spécifique à cette série d'expériences augm Qc+NP, à l'augmentation de la filtration transcapillaire au niveau de la circulation systémique sous l'effet de la vasodilatation liée au NP. CI/Cp a augmenté non significativement par rapport à sa valeur de contrôle soit $0,725 \pm 0,018$ vs $0,696 \pm 0,012$.

Les Figures 2 et 3 illustrent respectivement les variations individuelles de Q1 en fonction de la variation de Pc et des RVP.

La Figure 4 illustre les relations entre $\mathrm{Cl} / \mathrm{Cp}$ et l'augmentation relative de Q1 par rapport à sa valeur de contrôle (Q1/Q10) pendant l'état stable augm Qc + NP. Ces relations s'écartent de la zone ombrée (Figure 4) qui recouvre les relations observées au 
cours d'une augmentation de la pression capillaire pulmonaire $(\mathrm{Pc})$ pour une perméabilité capillaire normale en zone 3 .

En conclusion, l'augmentation de Qc associée à une vasodilatation par le NP entraîne une augmentation notable de Q1 et une légère élévation de $\mathrm{CI} / \mathrm{Cp}$, soit un tamisage des protéines inférieur à celui entraîné par une augmentation de Pc.

\section{Modifications hémodynamiques et lymphatiques induites par un stimulus hydrostatique (augmentation de Pog)}

Les valeurs moyennes du deuxième état stable des 4 expériences témoins où les shunts artério-veineux n'ont pas été ouverts, où le NP n'a pas été perfusé, mais où les préparations ont été soumises à un gonflement du ballonnet dans l'oreillette gauche (augm Pog) sont:

- pour les variables hémodynamiques : une élévation de la Pog de $11 \mathrm{~mm} \mathrm{Hg}$ (de 4,9 $\pm 1,0$ à 15,9 $\pm 1,3 \mathrm{~mm} \mathrm{Hg}, \mathrm{p}<0,001)$, une augmentation de Pap de 15,4 $\pm 1,0$ à 22,8 \pm $1,5 \mathrm{~mm} \mathrm{Hg}(\mathrm{p}<0,05)$, une élévation de Pc de $11,3 \pm 0,4$ à 19,9 $\pm 1,3 \mathrm{~mm} \mathrm{Hg}(\mathrm{p}<0,01)$, un Qc abaissé non significativement de 1,88 \pm 0,4 à $1,29 \pm 0,1$ 1/min et une diminution non significative des RVP.

- pour les variables lymphatiques : un presque doublement de $\mathrm{Ql}(\mathrm{de} 60 \pm 41$ à $11 \pm$ $33 \mu \mathrm{l} / \mathrm{min}, \mathrm{p}<0,05)$, une diminution de $\mathrm{Cl} / \mathrm{Cp}$ (de 0,736 $\pm 0,038$ à $0,630 \pm 0,035, \mathrm{p}<0,01)$.

En conclusion, ces résultats montrent que la préparation expérimentale en ellemême n'augmente pas la perméabilité des capillaires pulmonaires.

Les valeurs moyennes du troisième état stable des 3 expériences augm $\mathrm{Qc}+\mathrm{Np}$, où un état stable supplémentaire avec augmentation de Pog a été réalisée (Figure 1) sont, par comparaison à l'état stable de contrôle :

- pour les variables hémodynamiques : une élévation de la Pog de $17 \mathrm{~mm} \mathrm{Hg}$ (de 6,3 $\pm 1,9$ à 23,3 $\pm 1,7 \mathrm{~mm} \mathrm{Hg}, \mathrm{p}<0,05)$, une augmentation de Pap de $12 \mathrm{~mm} \mathrm{Hg}$ (de 19,7 \pm 2,1 à 32,7 $\pm 2,4 \mathrm{~mm} \mathrm{Hg}, \mathrm{p}<0,05)$, un Qc abaissé non significativement de $1,65 \pm 0,31$ à $1,29 \pm 0,21 / \mathrm{min}$.

- pour les variables lymphatiques : un Q1 multiplié par un facteur 8 (de 13,4 $\pm 2,1$ à $113,2 \pm 49,2 \mu 1 / \mathrm{min})$, une diminution de $\mathrm{Cl} / \mathrm{Cp}$ de $0,698 \pm 0,024$ à $0,542 \pm 0,031, \mathrm{p}<$ $0,05)$.

La Figure 4 illustre les relations de $\mathrm{Cl} / \mathrm{Cp}$ en fonction de l'augmentation relative de Q1 par rapport à sa valeur de contrôle $\left(\mathrm{Q} 1 / \mathrm{Ql}_{0}\right)$. Ces relations s'inscrivent dans la zone ombrée qui recouvre les relations observées au cours d'une augmentation de la pression capillaire pulmonaire $(\mathrm{Pc})$ pour une perméabilité capillaire normale en zone 3 .

En conclusion, ces résultats confirment que les parois capillaires n'ont pas subi de lésion durable pendant l'état stable de l'expérience où une augmentation du Qc était associée à une vasodilatation liée à la perfusion de NP.

\section{DISCUSSION}

Caractéristiques hémodynamiques et lymphatiques selon qu'elles sont liées à une augmentation de $\mathrm{Pc}$ ou à une augmentation de Qc

La caractérisation de la FCP secondaire à une augmentation de Pc a fait l'objet de nombreuses études dans les préparations expérimentales in vivo au moyen des mesures de Q1 et du rapport Cl/Cp. L'augmentation de Pc est le plus souvent obtenue en gênant le retour veineux pulmonaire par le gonflement partiel d'un ballonnet dans l'oreillette gauche. Il en résulte une augmentation de la Pog, qui entraîne une augmentation de Pc en amont. L'augmentation de Pc entraîne une telle augmentation du débit de FCP de liquide que le transport convectif des protéines qui 
Tableau 1: Effets de l'augmentation de débit cardiaque associée à la vas̄odilatation par le nitroprussiate (augm Qc + NP) sur les variables hémodynamiques et lymphatiques.

\begin{tabular}{|c|c|c|c|c|c|c|c|c|c|c|}
\hline Variables & $\begin{array}{c}\text { Pas } \\
\text { mmHg }\end{array}$ & $\begin{array}{c}\text { Pap } \\
\text { mmHg }\end{array}$ & $\begin{array}{c}\text { Pog } \\
\text { mmHg }\end{array}$ & $\begin{array}{c}\text { Pc } \\
\text { mmHg }\end{array}$ & $\begin{array}{c}\text { Qc } \\
\text { 1/min }\end{array}$ & $\begin{array}{c}\text { RVP } \\
\mathrm{mmHg} / \mathrm{l} / \mathrm{min}\end{array}$ & $\begin{array}{c}\text { Ql } \\
\mu \mathrm{l} / \mathrm{min}\end{array}$ & $\begin{array}{l}\mathrm{Cl} \\
\mathrm{g} / \mathrm{l}\end{array}$ & $\begin{array}{l}\text { Cp } \\
\text { g/l }\end{array}$ & $\mathrm{Cl} / \mathrm{Cp}$ \\
\hline \multirow{2}{*}{$\begin{array}{l}\text { Contrôle } \\
n=10\end{array}$} & 126 & 18,9 & 6,0 & 13.6 & 1,97 & 3,95 & 16,0 & 42,4 & 62,0 & 0,696 \\
\hline & 5 & 1,2 & 0,8 & 1,0 & 0,15 & 0,94 & 4,9 & 1,4 & 2,3 & 0,012 \\
\hline \multirow{2}{*}{$\begin{array}{l}\text { augm Qc+NP } 100 \\
\mathrm{n}=10\end{array}$} & & 20,6 & 6,1 & 15,5 & 2,84 & 3,10 & 30,1 & 39,60 & 54,5 & 0,725 \\
\hline & $3 *$ & 1,4 & 0,8 & $1,0 *$ & $0,3^{*}$ & $0,73^{*}$ & $7,5^{*}$ & 1,95 & $1,9 *$ & 0,018 \\
\hline
\end{tabular}

Valeurs moyennes des variables hémodynamiques et lymphatiques $\mathrm{m}+\mathrm{SE}$; $\mathrm{n}$, nombre d'expériences.

Pas, pression artérielle systémique; Pap, pression artérielle pulmonaire; Pog, pression de l'oreillette gauche; Pc, pression capillaire mesurée; Qc, débit cardiaque; $\mathrm{RVP}$, résistances vasculaires pulmonaires; $\mathrm{Ql}$, débit lymphatique; $\mathrm{Cl}$, concentration protéique de la lymphe; $\mathrm{Cp}$, concentration protéique plasmatique;

RVP, résistan

$\mathrm{Cl} / \mathrm{Cp}$, rappot de cardiaque as une analyse de variance; $(*) \mathrm{p}<0,05$

Tableau 2: Modifications des variables hémodynamiques et lymphatiques induites par une augmentation de la pression auriculaire gauche (augm.POG) succédant à une augmentation de débit cardiaque associée à une perfusion de nitroprussiate de sodium (augm Qc+NP).

\begin{tabular}{|c|c|c|c|c|c|c|}
\hline Variables & $\begin{array}{c}\text { Pas } \\
\text { mmHg }\end{array}$ & $\begin{array}{c}\text { Pap } \\
\text { mmHg }\end{array}$ & $\begin{array}{l}\text { Pog } \\
\text { mmHg }\end{array}$ & $\begin{array}{c}\text { Qc } \\
\text { l/min }\end{array}$ & $\begin{array}{c}\text { Ql } \\
\mu \mathrm{l} / \mathrm{min}\end{array}$ & $\mathrm{Cl} / \mathrm{Cp}$ \\
\hline \multirow{2}{*}{$\begin{array}{l}\text { Contrôle } \\
n=3\end{array}$} & 128 & 19,7 & 6,3 & 1,65 & 13,4 & 0,698 \\
\hline & 6 & 2,1 & 1,9 & 0,31 & 2,1 & 0,024 \\
\hline \multirow{2}{*}{$\begin{array}{l}\text { augm } Q c \pm N P \\
n=3\end{array}$} & 97 & 20 & 6,8 & 2,42 & 32,7 & 0,682 \\
\hline & 8* & 1,0 & 2,0 & 0,67 & 8,9 & 0,031 \\
\hline \multirow{2}{*}{$\begin{array}{l}\text { augm POG } \\
n=3\end{array}$} & 97 & 32,7 & 23,3 & 1,29 & 113,2 & 0,542 \\
\hline & $5 *$ & $2,4^{*}$ & $1,7 *$ & 0,20 & 49,2 & 0,031 \\
\hline
\end{tabular}

Valeurs moyennes des variables hémodynamiques et lymphatiques $\mathrm{m}+\mathrm{SE} ; \mathrm{n}$, nonbre dexpériences.

Pas, pression artérielle systémique; Pap, pression artérielle pulmonaire; Pog, pression de l'oreillette gauche; Pc, pression capillaire mesurée; Qc, débit cardiaque;

$\mathrm{Ql}$, débit lymphatique; $\mathrm{Cl} / \mathrm{Cp}$, rapport de la concentration protéique de la lymphe rapportée à celle du plasma. Les valeurs recueillies au cours de l'état stable

d'augmentation de débit cardiaque associée à une vasodilatation par le nitroprussiate (augm Qc + NP) ont été comparées pour toutes les variables à celles de l'état stable de contrôle par une analyse de variance; $(*) \mathrm{p}<0,05$. 


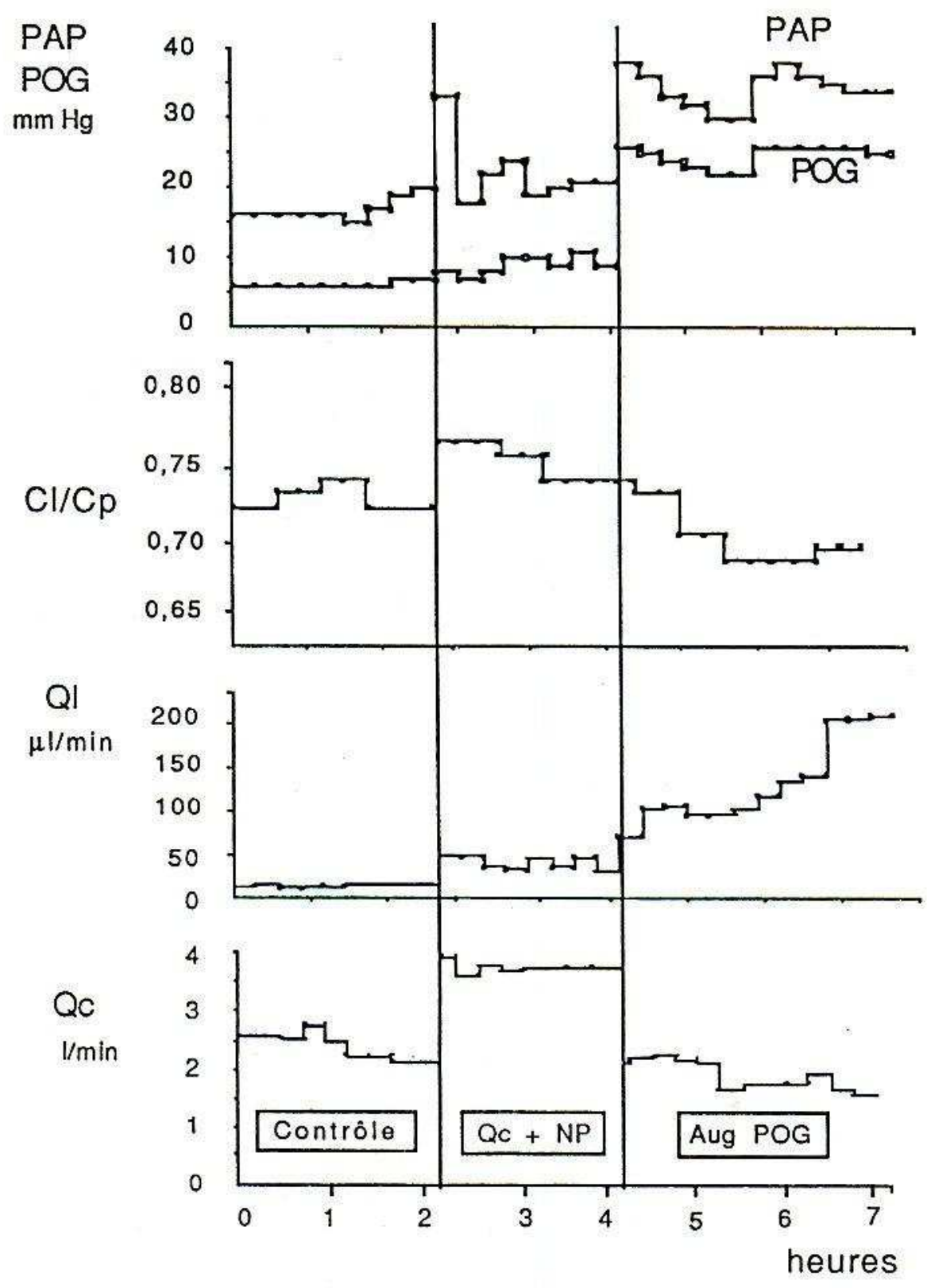

Figure 1: Elle illustre une expérience typique de cette étude avec un $1^{\text {er }}$ état stable de contrôle pendant 2 heures, un $2^{\text {ème }}$ état stable avec augmentation du débit cardiaque par ouverture de shunts artério-veineux associée à une vasodilatation au nitroprussiate de sodium pendant 2 heures, suivie d'un $3^{\text {ème }}$ état stable de 3 heures où la pression auriculaire gauche est augmentée alors que les shunts sont fermés et que la vasodilatation est arrêtée. 
QI $\mu 1 / \min$.

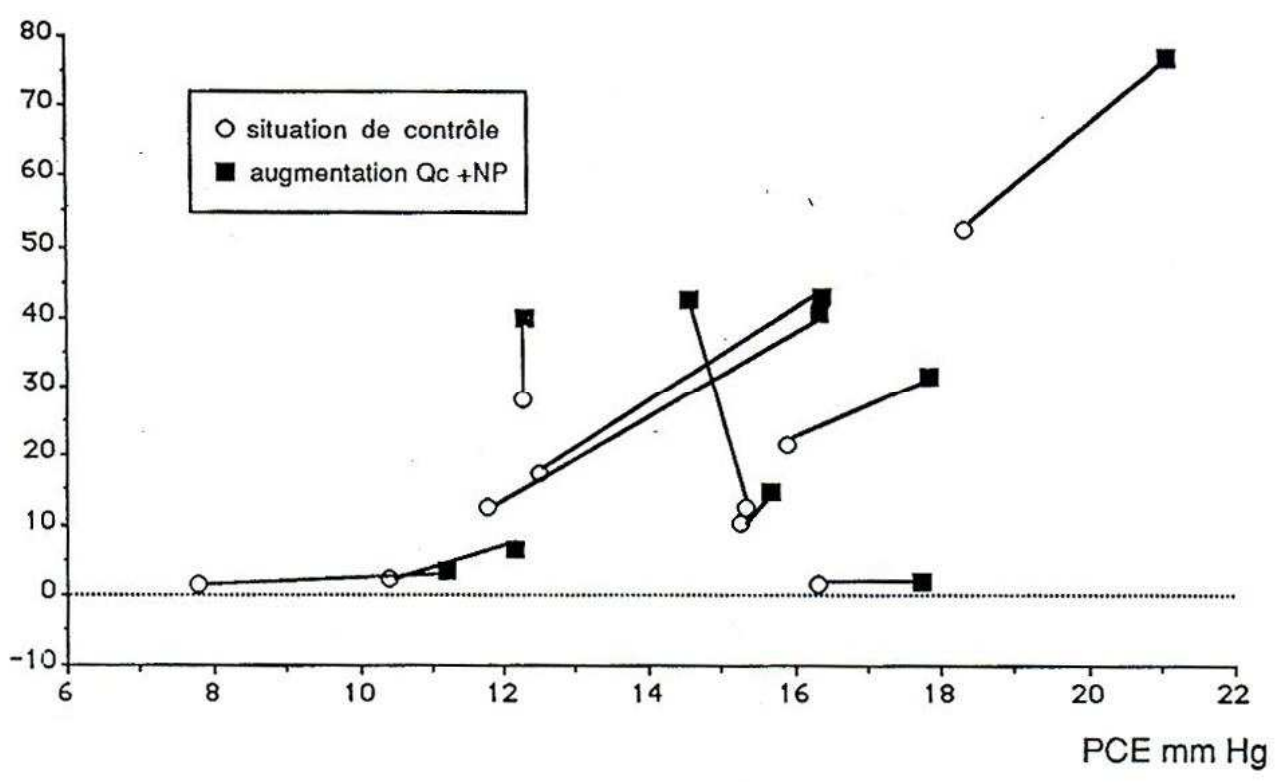

Figure 2 : Evolutions individuelles des débits lymphatiques Q1 en fonction des pressions capillaires pulmonaires $(\mathrm{Pc})$. Les ronds blancs correspondent à la situation de contrôle et les carrés noirs à l'augmentation de débit cardiaque (augm Qc) associée à une vasodilatation par le nitroprussiate de sodium (NP).

QI $(\mu / / \mathrm{min})$

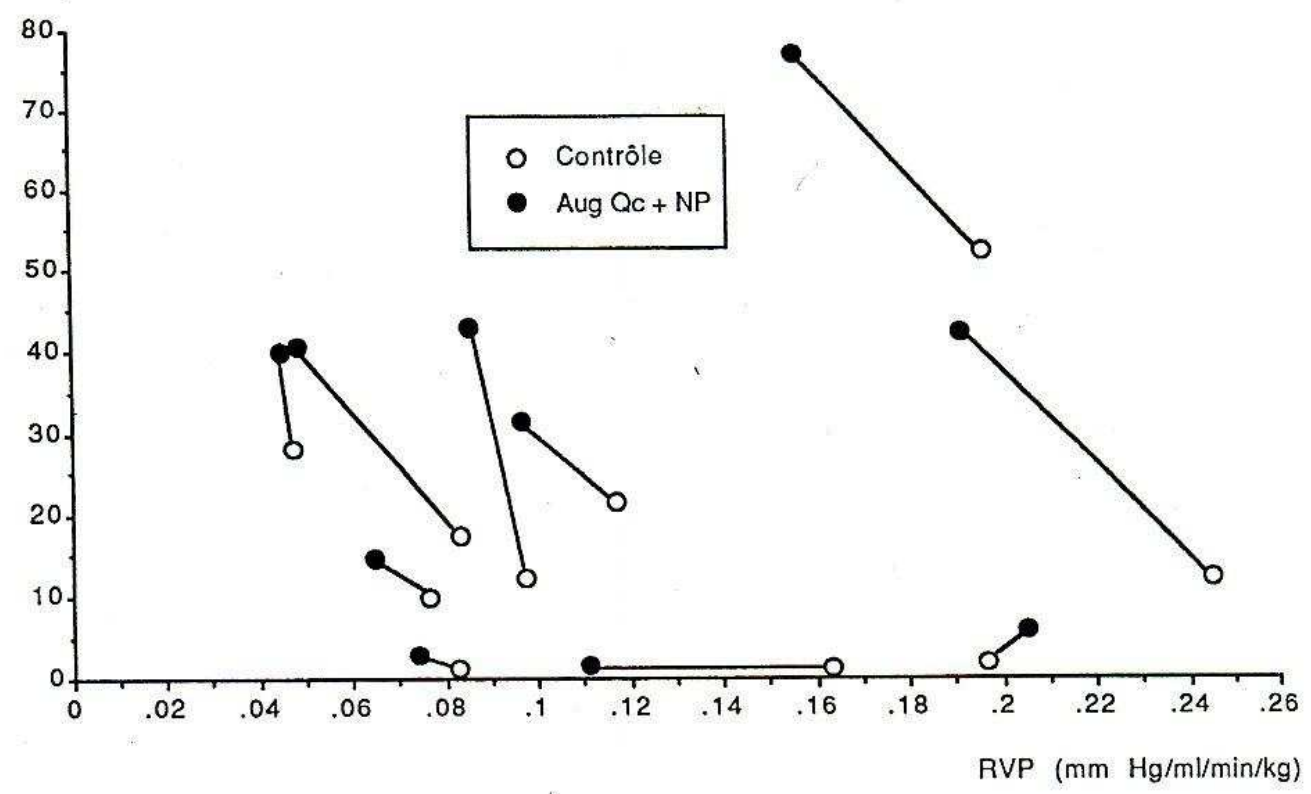

Figure 3 : Modifications des débits lymphatiques (Q1) en fonction des modifications des résistances vasculaires pulmonaires (RVP) induites par les augmentations de débit cardiaque (augm Qc) associées à une vasodilatation par le nitroprussiate de sodium (NP). 


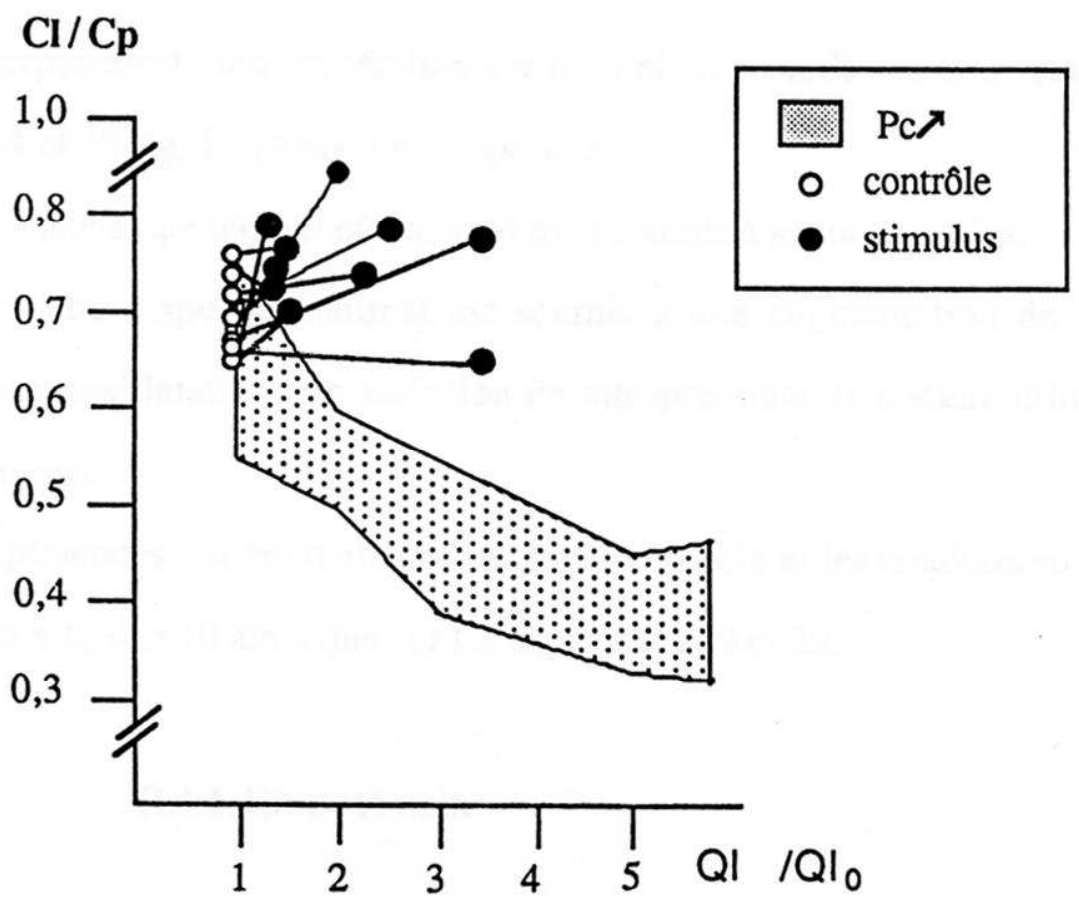

Figure 4: Rapports lymphe/plasma des concentrations en protéines $(\mathrm{Cl} / \mathrm{Cp})$ en fonction de l'augmentation du débit lymphatique relativement à sa valeur de contrôle (Q1/Q10).

Les ronds blancs correspondent à la situation de contrôle et les ronds noirs à l'augmentation de débit cardiaque associée à une vasodilatation par le nitroprussiate de sodium (augm Qc + NP).

La zone ombrée recouvre les relations observées au cours d'une augmentation de la pression capillaire pulmonaire (Pc) pour une perméabilité capillaire normale en zone 3. Les relations observées pour une augmentation isolée de débit cardiaque (augm Qc) restent dans cette zone (16). Les relations observées lorsqu'une vasodilatation par le nitroprussiate de sodium est associée à l'augmentation de $\mathrm{Qc}$ (stimulus ou augm Qc + NP) s'écartent de cette zone.

l'accompagne l'emporte sur le transport diffusif. De plus, le volume du compartiment interstitiel accessible aux protéines s'accroît, entraînant une dilution de celles-ci.

Q1 qui draine l'interstitium augmente et $\mathrm{Cl} / \mathrm{Cp}$ diminue aussi bien chez le mouton (Erdmann et al., 1975), que chez le chien (tewart et al., 2004). Cette méthode de référence entraîne cependant une gêne au retour veineux pulmonaire qui abaisse Qc et diminue la différence entre Pap et Pog.

Ces conditions expérimentales sont très différentes de celles qui prévalent dans des conditions physiologiques telles que l'exercice où Qc augmente. En effet, si une augmentation de Pog provoque, tout au long de l'arbre vasculaire pulmonaire, une augmentation homogène des pressions, une élévation physiologique de Qc augmente essentiellement la Pap. L'augmentation de Pap peut modifier les caractéristiques de la FCP même si la pression aérienne alvéolaire n'intervient pas sur le recrutement capillaire (conditions de zone 3 avec Pap > Pog > P aérienne alvéolaire) (West et al., 1964). Ces caractéristiques de la FCP peuvent changer selon que des différences apparaissent dans la distribution longitudinale de la pression, de la 
perméabilité ou de la surface d'échange. Une augmentation de la surface d'échange capillaire pourrait ainsi survenir à la suite d'une élévation de Pc, d'une élévation de Qc, d'une diminution des RVP, d'une élévation du volume sanguin pulmonaire avec distension et/ou recrutement de nouveaux capillaires.

Contrairement à l'augmentation de Pog qui entraîne une distension de la microcirculation pulmonaire, l'augmentation de Qc entraîne un recrutement capillaire comme le montre l'augmentation de la compliance de la circulation pulmonaire (Anglande et al., 1998) ou l'augmentation de la surface capillaire évaluée par l'activité pulmonaire de l'enzyme de conversion de l'angiotensine (Dupuis et al., 1992). L'augmentation de FCP induite par recrutement de nouveaux capillaires dont les capacités de tamisage protéique seraient similaires, devrait augmenter Q1, mais ne devrait pas modifier $\mathrm{Cl}$, ainsi $\mathrm{Cl} / \mathrm{Cp}$ resterait inchangé. Les deux conditions hémodynamiques que sont l'élévation de Pog et l'augmentation de Qc peuvent donc entraîner des modifications des caractéristiques de filtrations différentes malgré des augmentations identiques de Pc (Anglande et al., 1998). La modification du $\mathrm{Cl} / \mathrm{Cp}$ permet d'approcher le changement des caractéristiques de la FCP qui passent du domaine de l'augmentation de Pc à celle d'une augmentation de la surface d'échange.

\section{Caractéristiques hémodynamiques et lymphatiques liées à l'augmentation de Qc selon qu'elle est mécanique ou liée à l'exercice}

Les augmentations de Qc peuvent entraîner des caractéristiques différentes de FCP sur le poumon normal perfusé en zone 3 selon que l'augmentation de Qc est obtenue mécaniquement ou par l'exercice.

Les études rapportant les effets d'une augmentation mécanique de débit cardiaque sur les caractéristiques de la FCP utilisent plusieurs techniques pour augmenter le débit sanguin pulmonaire (pneumonectomie associée à une transfusion) (Landolt et al., 1983), circulation extra-corporelle de l'oreillette gauche à l'oreillette droite (Grimbert et al., 1988), ou création d'un canal artériel chez l'agneau (Feltes et al., 1989).

Ces études donnent des résultats assez homogènes sur les caractéristiques de la FCP quelles que soient les techniques utilisées pour augmenter mécaniquement Qc. Il est observé une augmentation de Q1 avec abaissement du rapport $\mathrm{Cl} / \mathrm{Cp}$, avec un tamisage des protéines similaire à celui observé lorsque l'on augmente Pc par élévation de la Pog.

Les études rapportant les effets d'une augmentation du débit cardiaque à l'exercice sur les caractéristiques de la FCP font état de résultats plus variés. Tantôt les caractéristiques de la FCP se rapprochent de celles observées au cours d'une augmentation de Pc par élévation de Pog, c'est-à-dire une augmentation de Q1 associée à un abaissement de Cl/Cp (Newman et al., 1988 ; Newman et al., 1983). Tantôt les caractéristiques de la filtration se rapprochent de celles observées au cours d'une augmentation de la surface d'échange avec une augmentation de Q1 associé à un abaissement du rapport $\mathrm{Cl} / \mathrm{Cp}$ faible ou absent (Coates et al., 1984; Koizumi et al., 2001; O'brodowich et al., 1986). Tantôt, les résultats sont en faveur de la coexistence de ces deux mécanismes (Coates et al., 1993). La surface d'échange capillaire peut être mesurée par d'autres techniques telles que l'extraction capillaire pulmonaire de la noradrénaline ou l'activité pulmonaire de l'enzyme de conversion de l'angiotensine. Le produit perméabilité-surface est multiplié par 3 chez le chien à l'exercice alors que la perméabilité capillaire pulmonaire est supposée normale. Ceci témoigne d'une surface d'échange multipliée par 3 pour un Qc multiplié par 3 (Dupuis et al., 1990, 1992). 
Hypothèse sur le mécanisme des caractéristiques hémodynamiques et lymphatiques pulmonaires à l'exercice selon l'importance du tonus vasculaire pulmonaire

Certaines formes d'adaptation à l'exercice de la circulation pulmonaire normale en zone 3 pourraient être assimilables à un modèle simple, celui d'une augmentation isolée de Qc avec distension mécanique des vaisseaux pulmonaires à chaque augmentation de la pression transmurale. Ce modèle simple d'augmentation isolée du débit cardiaque se traduirait par des caractéristiques de FCP proches de celles entraînées par une augmentation de Pc (augmentation de Q1 avec abaissement du rapport $\mathrm{Cl} / \mathrm{Cp}$ ). Néanmoins, les modèles plus complexes d'adaptation de la circulation pulmonaire à l'exercice n'excluent pas le recrutement au niveau des capillaires pulmonaires et l'intervention de facteurs neuro-humoraux (Reeves et al., 1996). Un modèle hémodynamique plus complexe pourrait s'appliquer aux caractéristiques de la filtration transcapillaire pulmonaire présentes au cours d'une augmentation de la surface d'échanges (augmentation de Ql avec maintien du rapport $\mathrm{Cl} / \mathrm{Cp}$ ). L'augmentation de débit cardiaque liée à l'exercice pourrait alors être associée à un abaissement du tonus vasculaire pulmonaire.

L'hypothèse d'une libération d'oxyde nitrique (NO) par l'endothelium stimulé par l'augmentation de Qc pourrait rendre compte de l'abaissement du tonus vasculaire pulmonaire à l'exercice. Cette hypothèse a reçu une confirmation expérimentale chez le mouton (Kane et al., 1984), mais chez le chien, le NO ne contribue pas à la régulation $\mathrm{du}$ tonus vasculaire pulmonaire de base (Cremona et al., 1994). En revanche, une perfusion de NP, qui libère pharmacologiquement du NO, entraine une vasodilatation pulmonaire significative chez le chien (Corboz et al., 1995; Kadowitz et al.,
1981). De plus, elle diminue sans abolir les pressions critiques de fermeture des vaisseaux pulmonaires (Mayers et al., 1990).

La canulation lymphatique chez l'animal éveillé n'a pas été développée chez le chien et il n'était donc pas possible de mesurer les caractéristiques de la FCP à l'exercice chez cet animal.

Nous avons néanmoins souhaité explorer l'hypothèse selon laquelle l'association d'un abaissement $\mathrm{du}$ tonus vasculaire pulmonaire à une augmentation mécanique du débit cardiaque rapprocherait les caractéristiques de la FCP de celles d'une augmentation du débit cardiaque provoquée par l'exercice (augmentation de Q1 avec abaissement faible ou absent du rapport $\mathrm{Cl} / \mathrm{Cp}$ ). Nous avons donc associé chez le chien une vasodilatation pharmacologique au NP à une augmentation mécanique du débit cardiaque. Nous avons alors comparé les caractéristiques de la FCP obtenues à celles d'une augmentation mécanique du débit cardiaque obtenues dans une étude précédente dans notre laboratoire (Grimbert et al., 1988). Enfin, nous avons vérifié si les résultats des expériences (augm Qc $+\mathrm{NP}$ ) se rapprochaient davantage que ceux des expériences augm Qc de ceux des études ayant mesuré la FCP à l'exercice.

\section{Modifications hémodynamiques et de QI liées à la perfusion de NP associée à l'augmentation mécanique du Qc dans cette étude}

Qc a augmenté significativement mais moins que dans l'étude précédente. Pc a augmenté significativement dans les deux études. En revanche, la Pap n'a pas augmenté significativement contrairement à l'étude précédente. En effet, la perfusion de NP a entraîné une vasodilatation pulmonaire avec une baisse des RVP qui n'était pas observée dans l'étude précédente. Ces modifications hémodynamiques des expériences augm Qc + 
NP et augm Qc sont similaires à celles observées chez le chien dont les poumons lésés par l'acide oléique avaient été soumis à de mêmes augmentations de débit cardiaque, soit mécaniques (ouverture de shunts artérioveineux), soit pharmacologiques (perfusion de NP) (Angle et al., 1984).

Q1 qui correspond à la FCP a augmenté significativement dans les expériences (augm $\mathrm{Qc}+\mathrm{NP}$ ), en fait davantage que dans l'étude précédente (augm Qc) (Grimbert et al., 1988). Cette augmentation de Q1 peut être attribuée en partie au moins à l'augmentation du Qc. En effet, dans les 7 expériences sur 10 où les variations de $\mathrm{Pc}$ sont supérieures à $1 \mathrm{~mm} \mathrm{Hg}$ (Figure 2), la pente d'augmentation de Ql est trois fois supérieure à celle observée pour une augmentation isolée de Qc dans notre étude précédente (Grimbert et al., 1988). La pente d'augmentation de Q1 est également trois fois supérieure à celle observée pour une augmentation de Pog dans le même type de préparation expérimentale (Parker et al., 1981) ou pendant les augmentations de Pog dans les expériences témoins et dans le $3^{\mathrm{è}}$ état stable des expériences augm Qc + NP.

Nous mettons en relation cette forte augmentation de Q1 dans les expériences augm Qc + NP avec la diminution des RVP dans 9 de ces expériences (Figure 3). En effet, cette diminution des RVP n'était pas présente dans les expériences augm Qc de l'étude précédente (Grimbert et al., 1988). Cette forte augmentation de Q1 pourrait être attribuée à un recrutement et à une augmentation de la surface d'échanges, mais cette interprétation est prématurée avant d'avoir examiné les caractéristiques de filtration des protéines.

\section{Modifications des variables lymphatiques} attribuables à la préparation expérimentale

La pente d'augmentation de Q1 trois fois supérieure dans les expériences augm Qc + NP à celle observée dans les expériences augm Qc dans notre étude précédente aurait pu s'expliquer par une différence de perfusion zonale entre les préparations expérimentales. Mais cette explication nous paraît peu vraisemblable, toutes les expériences ayant été pratiquées dans la même position latérale et donc probablement en zone 3 (Pap > Pog > P aérienne alvéolaire) (West et al., 1964).

Les caractéristiques de la filtration capillaire pulmonaire observées au cours de nos expériences (augmentation de Q1 avec abaissement non significatif du rapport $\mathrm{Cl} / \mathrm{Cp}$ ) sont celles d'une augmentation de la surface d'échange, mais elles pourraient être confondues avec celles d'une augmentation de perméabilité, notamment pour de faibles augmentations de Q1 (Talor et al., 1984). Nous avons donc contrôlé la normalité de la perméabilité capillaire pulmonaire dans nos préparations expérimentales en effectuant un tamisage des protéines à l'aide d'une élévation de Pog au cours de deux séries expérimentales.

La première série visait à vérifier si l'installation des shunts artério-veineux dans nos préparations expérimentales n'entraînait pas une augmentation de la perméabilité capillaire pulmonaire, même quand ces shunts étaient fermés. Les résultats d'une série de contrôle $(n=4)$ exposés en début de paragraphe 3.2 montrent un abaissement du rapport $\mathrm{CI} / \mathrm{Cp}$ en fonction de l'accroissement de Q1, qui est conforme à la norme habituelle dans notre préparation. Il n'y a donc pas d'augmentation de perméabilité avant l'augmentation de Qc et la perfusion de NP.

La deuxième série expérimentale $(\mathrm{n}=3)$ visait à contrôler au cours d'un 3ème état stable dans la série principale que l'augmentation de Qc et la perfusion de NP n'avaient pas modifié la perméabilité capillaire de la préparation. Après avoir atteint un état stable des variables lymphatiques pendant l'augmentation de Qc et la perfusion de NP, les shunts artério-veineux étaient 
fermés et la perfusion de NP arrêtée avant d'élever la Pog (Fig. 1). Les résultats exposés au Tableau 2 montrent un abaissement du rapport $\mathrm{CI} / \mathrm{Cp}$ en fonction de l'accroissement de Q1 qui est conforme à la norme habituelle dans notre préparation. Il n'y a donc pas d'augmentation de perméabilité après l'augmentation de Qc et la perfusion de NP.

\section{Modifications des variables lymphatiques liées à la perfusion de NP associée à l'augmentation mécanique du Qc}

Le rapport $\mathrm{Cl} / \mathrm{Cp}$ moyen ne varie pas significativement dans les expériences augm Qc + NP alors que Q1 moyen double (voir Tableau 1). La Figure 4 illustre les variations individuelles de $\mathrm{C} / \mathrm{Cp}$ en fonction des variations individuelles de Ql dans les expériences augm Qc + NP. Ces variations sortent de la zone où elles sont habituellement confinées lorsqu'elles sont mesurées après élévation de Pog dans les expériences témoins de cette étude ou après augmentation de Qc dans notre étude précédente (Grimbert et al., 1988).

Ces relations de $\mathrm{Cl} / \mathrm{Cp}$ en fonction de Q1 devraient résulter d'une augmentation de la surface de filtration sous l'effet de la perfusion de NP pour autant qu'une augmentation de perméabilité soit totalement écartée, qu'elle soit liée à la préparation expérimentale, ce que nous avons confirmé (cf. paragraphe 4.5) ou qu'elle soit liée à un effet spécifique du NP pendant sa perfusion. Cet effet spécifique du NP pourrait être lié à une toxicité directe du NP sur l'endothelium capillaire. Il n'était pas prévu d'étudier spécifiquement l'effet du NP sur la perméabilité capillaire dans cette étude et nous ne pouvons pas apporter d'arguments pour ou contre cette possibilité. Néanmoins, la littérature ne signale pas d'augmentation directe de la perméabilité capillaire par le NP, mais plutôt une légère augmentation de la surface d'échange (Foulke et al., 1984). Les possibilités de recrutement capillaire par les vasodilatateurs sont faibles (Rayers et al., 1990).

Néanmoins, la perfusion de NP pourrait avoir recruté des capillaires dont la pression critique de fermeture était plus élevée en raison d'une vasoconstriction locale inflammatoire ou hypoxique.

Nous n'avons pas relevé dans la littérature $d$ 'effets du NP tendant à recruter des zones pulmonaires lésées inflammatoires dont la perméabilité capillaire serait augmentée (Huval et al., 1998; Johnson et al., 1989; Schuter et al., 1990). En revanche, les données de certaines études confirment que la vasoconstriction hypoxique locale qui protège les zones hypoventilées de la perfusion et qui améliore les échanges gazeux pulmonaires peut être altérée par la perfusion de NP (Colley et al., 1997). Certes, la perfusion de NP n'a pas modifié la $\mathrm{PaO} 2$ dans nos expériences, mais nos résultats ne permettent pas d'écarter totalement la participation d'une augmentation de la perméabilité liée à l'augmentation de la surface d'échanges, ceci d'autant que la différence de leurs caractéristiques de tamisage des protéines $(\mathrm{Cl} / \mathrm{Cp})$ sont difficiles à distinguer pour de faibles augmentations de Q1 (Taylor et al., 1984). Ainsi, les facteurs propres à notre protocole expérimental ont pu entraîner une surestimation de $\mathrm{Cl} / \mathrm{Cp}$, mais cette surestimation est modeste.

En conclusion, l'association d'une perfusion de NP qui libère du NO à l'augmentation mécanique de Qc réalisée dans cette étude permet de rapprocher les caractéristiques de FCP observées pendant les augmentations mécaniques de Qc (Feltes et al., 1989; Grimbert et al., 1988; Landolt et al., 1983) de celles observées pendant les augmentations de Qc à l'exercice (Coates et al., 1984, 1993; Koizumi et al., 2001; Newman et al., 1988 ; Newman et al., 1983; O'brodowich et al., 1986). Nous n'avons pas 
connaissance d'une autre étude qui ait cherché à explorer la possibilité de rapprocher les caractéristiques de FCP de ces deux types d'augmentation de Qc par l'association d'une vasodilatation. $\mathrm{Ce}$ rapprochement est en faveur d'un recrutement et d'une augmentation de la surface d'échange capillaire pulmonaire liée à la production de NO (Corboz et al., 1995; Kadowitz et al., 1981; Kane et al., 1984; Newman et al., 1990) par l'endothelium capillaire pulmonaire stimulé par l'augmentation du débit sanguin pulmonaire pendant l'exercice. Par ailleurs, les résultats de cette étude présentent l'intérêt de constituer des données de contrôle pour évaluer les effets d'une élévation de débit cardiaque sur la FCP dans le contexte d'une augmentation pathologique de la perméabilité capillaire, avec ses conséquences sur la thérapeutique des oedèmes pulmonaires de perméabilité en soins intensifs (Briot et al., 2009 ; Stewart et al., 2007).

\section{BIBLIOGRAPHIE}

Anglade D, Corboz M, Menaouar A, Parker JC, Sanou S, Bayat S, Benchetrit G, Grimbert FA. 1998. Blood flow vs. venous pressure effects on filtration coefficient in oleic acid-injured lung. $J$. Appl. Physiol., 84(3): 1011-1023.

Angle M, Ducas J, Schick U, Girling L, Prewitt RM. 1984. Direct effects of nitroprusside do not alter gas exchange in canine oleic acid edema. J. Appl. Physiol., 57(5): 1498-1501.

Briot R, Bayat S, Anglade D, Martiel JL, Grimbert F. 2009. Increased cardiac index due to terbutaline treatment aggravates capillary-alveolar macromolecular leakage in oleic acid lung injury in dogs. Crit. Care, 13(5): 166.

Broe PJ, Toung TJ, Permutt S, Cameron JL. 1983. Aspiration pneumonia: treatment with pulmonary vasodilators. Surgery,
94(1): 95-99.

Coates G, O'Brodovich H, Jefferies AL, Gray GW. 1984. Effects of exercise on lung lymph flow in sheep and goats during normoxia and hypoxia. J. Clin. Invest., 74(1): 133-141.

Coates G, O'Brodovich H, Goeree G. 1993. Hindlimb and lung lymph flows during prolonged exercise. J. Appl. Physiol., 75(2): 633-638.

Colley PS, Cheney FW. 1977. Sodium nitroprusside increases Qs/Qt in dogs with regional atelectasis. Anesthesiology, 47(4): 338-341.

Corboz M, Sanou S, Grimbert FA. 1995. Capillary pressure estimates from arterial and venous occlusion in intact dog lung. Eur. Respir. J., 8(7): 1122-1129.

Cremona G, Wood AM, Hall LW, Bower EA, Higenbottam T. 1994. Effect of inhibitors of nitric oxide release and action on vascular tone in isolated lungs of pig, sheep, dog and man. $J$. Physiol.,15(481): 185-195.

Dupuis J, Goresky CA, Juneau C, Calderone A, Rouleau JL, Rose CP, Goresky S. 1990. Use of norepinephrine uptake to measure lung capillary recruitment with exercise. J. Appl. Physiol., 68(2): 700713.

Dupuis J, Goresky CA, Ryan JW, Rouleau JL, Bach GG. 1992. Pulmonary angiotensinconverting enzyme substrate hydrolysis during exercise. J. Appl. Physiol., 72(5): 1868-1886.

Erdmann AJ 3rd, Vaughan TR Jr, Brigham KL, Woolverton WC, Staub NC. 1975. Effect of increased vascular pressure on lung fluid balance in unanesthetized sheep. Circ. Res., 37(3): 271-284.

Feltes TF, Hansen TN. 1989. Effects of an aorticopulmonary shunt on lung fluid balance in the young lamb. Pediatr. Res., 26(2): 94-97.

Foulke GE, Fisher CJ Jr, Demling RH. 1984. 
Effect of nitroprusside administration and sudden withdrawal on pulmonary vascular tone and lung fluid balance. Crit. Care Med., 12(5): 432-435.

Grimbert FA, Parker JC, Taylor AE. 1981. Increased pulmonary vascular permeability following acid aspiration. $J$. Appl. Physiol., 51(2): 335-345.

Grimbert FA, Martin D, Parker JC, Taylor AE. 1988. Lymph flow during increases in pulmonary blood flow and microvascular pressure in dogs. Am. J. Physiol., 255(5): 1149-1155.

Holloway H, Perry M, Downey J, Parker J, Taylor A. 1983. Estimation of effective pulmonary capillary pressure in intact lungs. J. Appl. Physiol., 54(3): 846-851.

Huval WV, Lelcuk S, Feingold H, Valeri CR, Shepro D, Hechtman HB. 1988. Effects of nitroprusside and ketanserin upon pulmonary edema after acid injury. Surg. Gynecol Obstet., 166(6): 527-534.

Johnston WE, Vinten-Johansen J, Tommasi E. 1989. Sodium nitroprusside and positive end-expiratory pressure are not detrimental in canine asymmetric pulmonary edema. Crit. Care Med., 17(3): 241-246.

Jonkam CC, Bansal K, Traber DL, Hamahata A, Maybauer MO, Maybauer DM, Cox RA, Lange M, Connelly RL, Traber LD, Djukom CD, Salsbury JR, Herndon DN, Enkhbaatar P. 2009. Pulmonary vascular permeability changes in an ovine model of methicillin-resistant Staphylococcus aureus sepsis. Crit. Care, 13(1): R19.

Kadowitz PJ, Nandiwada P, Gruetter CA, Ignarro LJ, Hyman AL. 1981. Pulmonary vasodilator responses to nitroprusside and nitroglycerin in the dog. J. Clin. Invest. Mar., 67(3): 893902.

Kane DW, Tesauro T, Koizumi T, Gupta R, Newman JH. 1994. Exercise-induced pulmonary vasoconstriction during combined blockade of nitric oxide synthase and beta adrenergic receptors. J. Clin. Invest., 93(2): 677-683.

Koizumi T, Roselli RJ, Parker RE, HermoWeiler CI, Banerjee M, Newman JH. 2001. Clearance of filtered fluid from the lung during exercise: role of hyperpnea. Am. J. Respir. Crit. Care Med., 163(3): 614-618.

Landolt CC, Matthay MA, Albertine KH, Roos PJ, Wiener-Kronish JP, Staub NC. 1983. Overperfusion, hypoxia, and increased pressure cause only hydrostatic pulmonary edema in anesthetized sheep. Circ. Res., 52(3): 335-341.

Mayers I, Johnson DH. 1990 .Vasodilators do not abolish pulmonary vascular critical closing pressure. Respir Physiol., 81(1): 63-73.

Newman JH, Butka BJ, Parker RE, Roselli RJ. 1988. Effect of progressive exercise on lung fluid balance in sheep. J. Appl. Physiol., 64(5): 2125-2131.

Newman JH, Cochran CP, Roselli RJ, Parker RE, King LS. 1993. Pressure and flow changes in the pulmonary circulation in exercising sheep: evidence for elevatedmicrovascular pressure. Am. Rev. Respir. Dis., 147(4): 921-926.

O'Brodovich H, Coates G. 1986. Effect of isoproterenol or exercise on pulmonary lymph flow and hemodynamics. J. Appl. Physiol., 60(1): 38-44.

Parker JC, Parker RE, Granger DN, Taylor AE. 1981. Vascular permeability and transvascular fluid and protein transport in the dog lung. Circ. Res., 48(4): 549561.

Reeves JT, Taylor AE. 1996. Pulmonary hemodynamics and fluid exchange in the lungs during exercise. In Handbook of Physiology. Exercise: Regulation and Integration of Multiple Systems. Bethesda, MD(ed). Am. Physiol. Soc., sect. 3; 587-613. 
Schreiber T, Hueter L, Gaser E, Schmidt B, Schwarzkopf K, Karzai W. 2007. Effects of a catecholamine-induced increase in cardiac output on lung injury after experimental unilateral pulmonary acid instillation. Crit. Care Med., 35: 17411748.

Schuster DP, Haller J. 1990. Regional pulmonary blood flow during acute pulmonary edema: a PE study. J. Appl. Physiol., 69(1): 353-361.

Stewart RH, Allen SJ, Quick CM, Rohn DA, Cox CS, Laine GA. 2004. Effect of venous air embolization on pulmonary microvascular protein permeability. Microcirculation, 11(5): 409-414.

Taylor AE, Granger DN. 1984. Exchange of macromolecules across the circulation. In Handbook of Physiology, The Cardiovascular System. The Microcirculation. Bethesda MD(ed). Am. Physiol. Soc., , sect. 2; 467-520.

West JB, Dollery CT, Naimark A. 1964 .Distribution od blood flow in isolated lung; relation to vascular and alveolar pressures. J. Appl. Physiol., 19: 713-724. 